\title{
MicroRNA-506-3p inhibits proliferation and promotes apoptosis in ovarian cancer cell via targeting SIRT1/AKT/FOXO3a signaling pathway
}

\author{
X. Y. XIA', Y. J. YU², F. YE, G. Y. PENG ${ }^{3}$, Y. J. LI' ${ }^{3}$ X. M. ZHOU ${ }^{4, *}$ \\ ${ }^{1}$ Scientific Research Department of Changsha Health Vocational College, Changsha, Hunan, China; ${ }^{2}$ Office of Changsha Health Vocational \\ College, Changsha, Hunan, China; ${ }^{3}$ Nursing College of Changsha Vocational College of Health, Changsha, Hunan, China; ${ }^{4}$ Internal Medicine- \\ Cardiovascular Department, The First Hospital of Hunan University of Chinese Medicine, Changsha, Hunan, China
}

*Correspondence: zxm6599@126.com; zhouxiaomingHUCM@yeah.net

Received May 17, 2019 / Accepted August 11, 2019

\begin{abstract}
Ovarian cancer (OC) is one of the most common tumors in females. Growing evidence shows that microRNA-506-3p (miR-506-3p) is downregulated in OC tissues. The purpose of this study was to investigate the mechanism of miR-506-3p in modulating OC. Quantitative reverse transcriptase PCR (qRT-PCR) was employed to investigate the expression of miR-506-3p and its target in OC tissues or cell lines. CCK-8 or colony formation assay was used to examine cell viability or proliferation, respectively. Flow cytometry was demonstrated to detect cell apoptosis. Western blot was then applied to analyze underlying mechanisms. The potential target of miR-506-3p was examined via luciferase reporter assay. MiR-506-3p was significantly downregulated in both human OC tissues and cell lines. Overexpression of miR-506-3p not only decreased cell viability of OC cell lines but also promoted cell apoptosis, thus inhibiting OC progression. Moreover, SIRT1 (Sirtuin 1) was found to be a direct target of miR-506-3p, and SIRT1 expression was negatively regulated by miR-506-3p in OC cell lines. Further investigation revealed that overexpression of SIRT1 could promote cell viability as well as inhibit cell apoptosis, showing the reversed effect on OC progression compared to miR-506-3p. Lastly, AKT (Protein kinase B) /FOXO3a (Forkhead box O3) signaling pathway was inactivated by miR-506-3p while activated by SIRT1, relating to regulation of miR-506-3p on OC progression. Our results revealed a novel mechanism by which miR-506-3p inhibited proliferation while promoted apoptosis of OC via inactivation of SIRT1/AKT/FOXO3a signaling pathway, suggesting that miR-506-3p might be a potential target for OC.
\end{abstract}

Key words: ovarian cancer, microRNA-506-3p, proliferation, apoptosis, SIRT1

Ovarian cancer (OC), as one of the most common tumors in females, remains a leading cause of morbidity and mortality [1]. Although the developing treatments including surgery, radiation therapy, and chemotherapy are widely used nowadays [2], the unsatisfactory five-year survival rate [3-5] urges researchers to find new therapeutic targets for OC. It has been reported that critical oncogenes or tumor suppressors have been suggested to be promising therapeutic targets for OC due to the regulation of OC development and progression [6,7]. However, the unclear pathogenesis and progression of OC suggests that it is urgent to study the molecular pathogenesis of OC metastasis and discover more effective therapeutic targets.

MicroRNAs (miRNAs) inhibit the gene expression via binding to the 3' untranslated regions (3'UTRs) of target mRNAs $[8,9]$. Growing evidences have shown that miRNAs are involved in the regulation of cell proliferation and apoptosis [10-12], and play an important role in the cancer cell progression $[13,14]$. A large number of miRNAs such as miR-21 [15], miR-181a [16], miR-506 [17], miR-199b-5p [18] have been found to be involved in regulation of OC. MiR-506-3p has been indicated to inhibit osteosarcoma [19], neural stem cell [20], esophageal squamous cell carcinoma [21] and non-small lung cancer [22] progression. However, the underlying mechanism of miR-506-3p in OC remains to be investigated.

SIRT1 (Sirtuin 1) belongs to member of the sirtuin proteins family and functions as deacetylase to regulate PGC1-alpha/ERR-alpha complex for the modulation of cell metabolism [23]. Moreover, SIRT1, as an intracellular regulatory protein, also deacetylates and thereby deactivates p53 [24] to regulate tumorigenesis. Recently, SIRT1 was found to be related with poor prognosis of epithelial ovarian cancer [25] and promoted ovarian cancer cell invasion [26] as an 
oncogene. However, the regulatory mechanism of SIRT1 on OC and the relationship between miR-506-3p/SIRT1 and OC are largely unknown. Therefore, the present study aimed to investigate the effects as well as the molecular mechanism of miR-506-3p on OC progression.

\section{Patients and methods}

Patients and tissues collection. Total of 20 normal healthy people and 60 patients diagnosed with primary, recurrent and metastatic OC $(n=20$ each) were recruited from the First Hospital of Hunan University of Chinese Medicine, Changsha, Hunan, China. Approval for this study was acquired from Ethics Committee of the First Hospital of Hunan University of Chinese Medicine. Before surgery, written informed consents were acquired from all participants. The tissues were harvested from patients immediately after surgical removal and kept in $-80^{\circ} \mathrm{C}$ freezer for the following experiments.

Cell culture. Normal human ovarian epithelial cell line (IOSE386), human embryonic kidney 293T (HEK-293T) cells and human OC cell lines HO-8910PM, A2780, HO-8910, CAOV3, SKOV3, OVCA433, PEO1 and COC1 were purchased from Chinese Academy of Sciences Committee on Type Culture Collection Cell Bank (Shanghai, China). All the cells were cultured in RPMI-1640 (Hyclone, Logan, UT, USA) containing $15 \%$ fetal bovine serum (GIBCO, Rockville, $\mathrm{MD}, \mathrm{USA}$ ) at incubator with $37^{\circ} \mathrm{C}$ and under a humidified atmosphere with $5 \% \mathrm{CO}_{2}$. The cells with confluence of $80 \%$ were used for the experiments.

Cell transfection. MiR-506-3p mimics, and the negative controls (miR-NC) were synthesized by GenePharma (Suzhou, China). For the overexpression of SIRT1, PCR was used to amplify SIRT1 and subcloned into expression plasmids pcDNA3.1 (Invitrogen, Carlsbad, CA, USA). HO-8910PM was seeded at a concentration of $4 \times 10^{5}$ cells per well in 12-well plates and incubated overnight. The culture medium was then removed and cells were washed with PBS. The cells were transfected with miR-506-3p mimics or miR-NC (40 nM), pcDNA3.1-SIRT1 or the empty vector via Lipofectamine 2000 (Invitrogen) according to the manufacturer's instructions. Cells were harvested for RNA or protein extraction and further analyses 48 hours after transfection.

qRT-PCR. Sample RNAs were extracted from OC tissues and cell lines via Trizol reagent (Invitrogen), and miRNAs were extracted using miRcute miRNA isolation kit (Tiangen, Beijing, China). The RNAs were then reverse transcribed into cDNAs using miScript Reverse Transcription kit (Qiagen, Hilden, Germany). cDNAs were amplified by using SYBR1 Premix Ex Taq ${ }^{\mathrm{TM}}$ II (Takara, Shiga, Japan). U6 was used as the internal reference and GAPDH as the endogenous controls. Three technological replicates were used to ensure the reliability of the analysis. The primer sequences were as shown: SIRT1, 5'-TAGCCTTGTCAGATAAGGAAGGA-3' (forward) and 5'-ACAGCTTCACAGTCAACTTTGT-3' (reverse); miR-506-3p, 5'-ACACTCCAGCTGGGTAAGGCACCCTTCTGA-3' (forward) and 5'-CTCAACTGGTGTCGTGGAGTCGGCAATTCAGTTGAGTCTACTCA-3' (reverse); U6, 5'-CTCGCTTCGGCAGCACA-3' (forward) and 5'-AACGCTTCACGAATTTGCGT-3' (reverse); GAPDH, 5'-TGTTCGTCATGGGTGTGAAC-3' (forward) and 5'-ATGGCATGGACTGTGGTCAT-3' (reverse). The relative expression was analyzed using the $2^{-\Delta \Delta \mathrm{Ct}}$ method [27].

Cell counting kit-8 (CCK-8) assay. Three thousand cells/ well transfected HO-8910PM cells were trypsinized and then cultured in 96-well plates for 24,48 or $72 \mathrm{~h}$. After removing the medium, the cells were washed with PBS. The CCK-8 assay (Beyotime Institute of Biotechnology, Haimen, China) was used to evaluate the cell viability at $490 \mathrm{~nm}$ by a spectrophotometer (BioTek, VT, USA).

Cloning formation assay. One million cells/ml transfected HO-8910PM cells were harvested. Then $4.1 \mathrm{ml}$ RPMI-1640 medium and $0.9 \mathrm{ml} 4 \%$ agar were warmed to $56^{\circ} \mathrm{C}$ and then cooled to precipitate. $3 \times 10^{4}$ cells $/ 3 \mathrm{ml}$ in RPMI- 1640 medium with $0.36 \%$ agar were diluted to form the single-cell suspension. All the colonies were stained with $0.04 \%$ crystal violet in $2 \%$ ethanol in PBS, and then incubated at $37^{\circ} \mathrm{C}$ for 3 weeks. The stained colonies were photographed under microscope.

Flow cytometry. $1 \times 10^{4}$ transfected HO-8910PM cells per well were harvested. The cells were then fixed with $70 \%$ ethanol at $4{ }^{\circ} \mathrm{C}$ for $30 \mathrm{~min}$. After washing with PBS, ribonuclease (Abcam, Cambridge, MA, USA) was added to the cells, and the propidium iodide (PI, $200 \mu \mathrm{l}$, Abcam) was used to stain the cells. FACS flow cytometer (Attune, Life Technologies, Darmstadt, Germany) was used to analyzed the cell cycle.

Bioinformatics analysis. TargetScan version 7.1 (www. targetscan.org) was utilized to predict the target genes of miR-506-3p.

Dual luciferase reporter assay. The fragments of wild type 3'UTR of SIRT1 (SIRT1-WT) containing the binding sites for miR-506-3p were amplified and cloned into psi-CHECK ${ }^{\mathrm{TM}}-2$ vector (Promega, Madison, WI, USA), as well as the mutant SIRT1 (SIRT1-MUT) whose binding ability with miR-506-3p were lost. $3 \times 10^{4} \mathrm{HEK}-293 \mathrm{~T}$ cells per well were seeded in 48-well plates for $24 \mathrm{~h}$. Cells were then transfected with psiCHECK $^{\mathrm{mm}}$-2-SIRT1-WT, psiCHECK ${ }^{\mathrm{sm}}$-2-SIRT1-MUT $(1 \mu \mathrm{g})$ in combination with miR-506-3p mimics (100 nM; GenePharma) via Lipofectamine 2000 (Invitrogen). 48 h later, luciferase activities were measured using the Dual Luciferase Reporter Assay System (Promega) and detected by Lumat LB 9501 luminator (EG\&G Berthold, Bundoora, Australia). Firefly luciferase activity was normalized to renilla luciferase activity for each group.

Western blot. Cultured HO-8910PM cells were harvested and lysed in RIPA buffer (KeyGen, Nanjin, China). Protein lysates were loaded onto $10 \%$ SDS-PAGE, and then transferred to PVDF membrane. The membrane was blocked in PBS-T with $5 \%$ BSA for $1 \mathrm{~h}$. PVDF membranes were then probed with rabbit anti-SIRT1 monoclonal antibody $(1: 1000$, 
ab32441, Abcam), AKT (1:500, ab8805, Abcam), p-AKT (1:1500, ab38449, Abcam), FOXO3a $(1 \mu \mathrm{g} / \mathrm{ml}, \mathrm{ab} 23683$, Abcam), p-FOXO3a (1:500, ab47285, Abcam), GAPDH (1:2500, ab9485, Abcam) overnight at $4^{\circ} \mathrm{C}$. The PVDF membrane was washed with TBST and labeled with horseradish peroxidase (HRP)-conjugated secondary antibodies (1:2000, ab6721, Abcam) for $1 \mathrm{~h}$. Immunoreactivities were detected by enhanced chemiluminescence (KeyGen). GAPDH was used as a control.

Statistical analysis. All results are expressed as mean \pm SEM of at least 3 independent experiments. By the means of GraphPad Prism software (GraphPad Prism Software Inc., San Diego, USA) and one-way analysis of variance (ANOVA), we determined the statistical analyses. A $\mathrm{p}<0.05, \mathrm{p}<0.01$ or $\mathrm{p}<0.001$ was considered as a mark of statistically significant.

\section{Results}

MiR-506-3p was downregulated in ovarian cancer. We firstly determined the expression level of miR-506-3p in OC. As shown in Figure 1A, the expression level of miR-506-3p was substantially decreased in 60 primary, recurrent and metastatic OC tissues compared with the normal tissues $(\mathrm{p}<0.001)$. Moreover, compared to the primary OC tissues, recurrent and metastatic OC tissues contained lower miR-506-3p expression (Figure 1A). To further confirm the downregulation of miR-506-3p in OC tissues, we then investigated the expression of miR-506-3p in several common ovarian cancer cell lines. As demonstrated in Figure 1B, miR-506-3p was also significantly decreased in ovarian cancer cell lines (HO-8910PM, A2780, HO-8910, CAOV3, SKOV3, OVCA433, PEO1 and COC1) compared with in the normal human ovarian epithelial cell line (IOSE386). Of those, HO-8910PM, as highly metastatic OC cell line, showed the lowest expression of miR-506-3p (Figure 1B) was selected for the following functional assays. Therefore, miR-506-3p was downregulated in both ovarian cancer tissues and cells, and the decreased expression level of miR-506-3p was associated with poor differentiation of OC.

MiR-506-3p inhibited cell proliferation and induced cell apoptosis of $\mathrm{OC}$. To investigate the functional role of miR-506-3p on OC, stable HO-8910PM cell line with overexpression of miR-506-3p was established via transfection with miR-506-3p mimics and confirmed in Figure 2A. Functional assays revealed that miR-506-3p overexpression not only decreased the cell viability (Figure $2 \mathrm{~B}$ ), but also decreased number of colonies of OC cells compared with the miR-NC group (Figures 2C and 2E). Moreover, flow cytometry showed that miR-506-3p overexpression promoted the OC cell apoptosis (Figures $2 \mathrm{D}$ and $2 \mathrm{~F}$ ). These results revealed that overexpression of miR-506-3p inhibited cell proliferation and induced cell apoptosis in OC.

SIRT1 is a direct target gene of miR-506-3p in OC. To uncover the underlying mechanism about miR-506-3p in OC, we firstly determined that SIRT1 was found to be a putative target for miR-506-3p via bioinformatics analysis (Figure 3A). Moreover, luciferase vectors containing wildtype (WT) or mutant (MUT) 3'UTR of SIRT1 were firstly constructed. Luciferase reporter assay was then employed and the result indicated that luciferase activity was significantly inhibited in HEK-293T cells co-transfected with the SIRT1-WT and miR-506-3p mimics, while no significantly changes were detected by co-transfection with the SIRT1-MUT and miR-506-3p mimic (Figure 3B). In general,
A

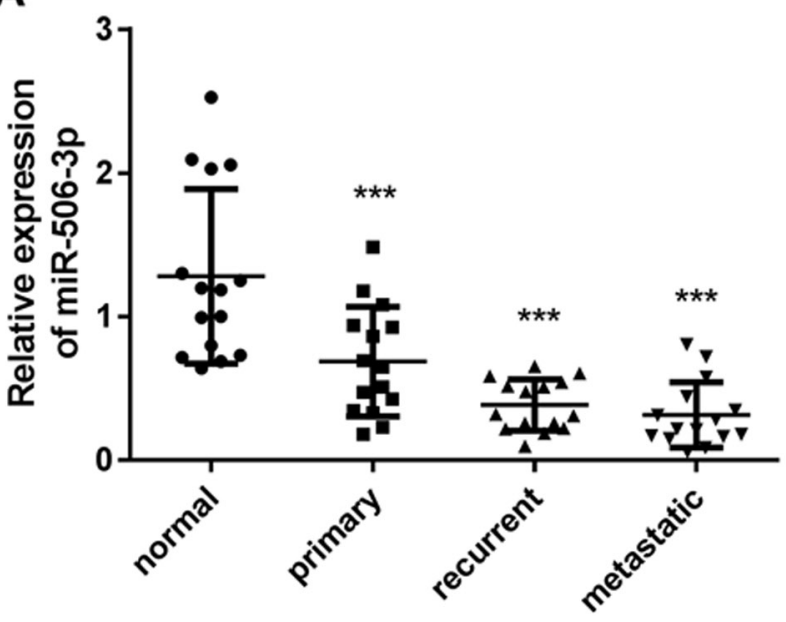

B

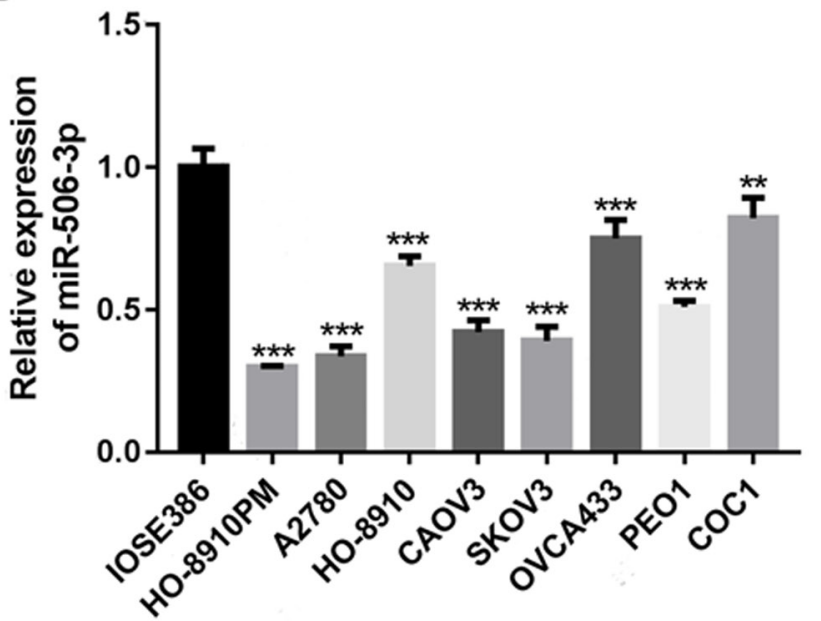

Figure 1. MiR-506-3p was downregulated in ovarian cancer. A) qRT-PCR analysis of miR-506-3p in human primary, recurrent and metastatic OC tissues $\left(n=45,15\right.$ each) and normal ovarian tissues $(n=15$, normal $) .{ }^{\star *}$ represents primary, recurrent and metastatic $0 C$ tissues vs. normal, $p<0.001$. $\left.B\right)$ qRT-PCR analysis of miR-506-3p in OC cell lines (HO-8910PM, A2780, HO-8910, CAOV3, SKOV3, OVCA433, PEO1 and COC1) and normal human ovarian epithelial cell line (IOSE386). ${ }^{* *},{ }^{* * *}$ represents vs. IOSE386, $\mathbf{p}<0.01, \mathrm{p}<0.001$. 
A

C

D

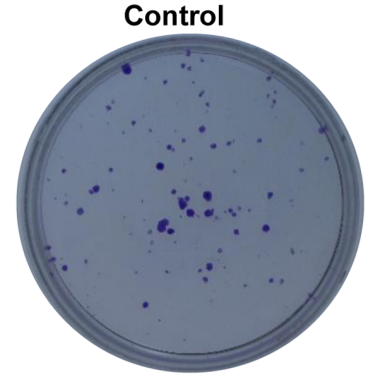

Control

Control
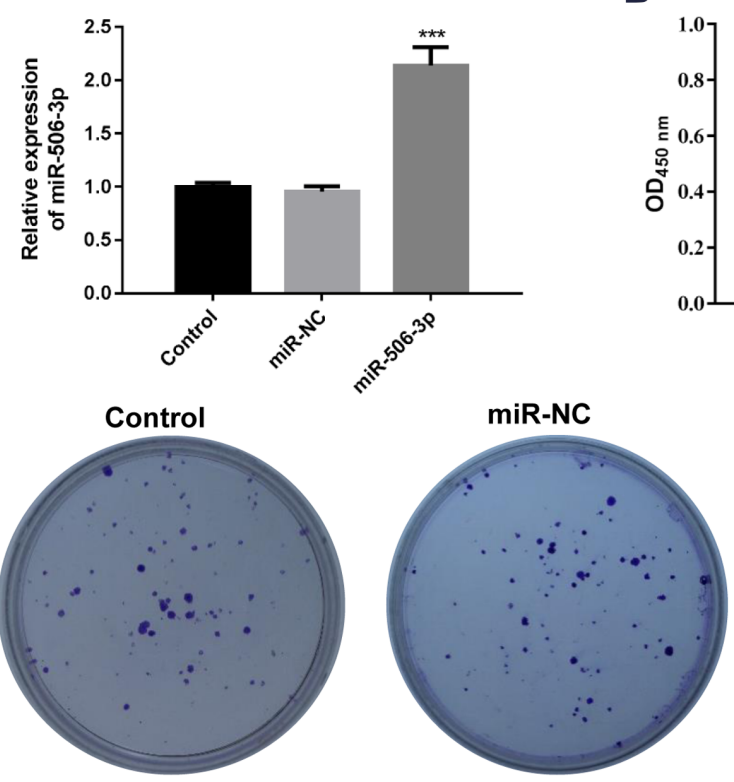

miR-NC

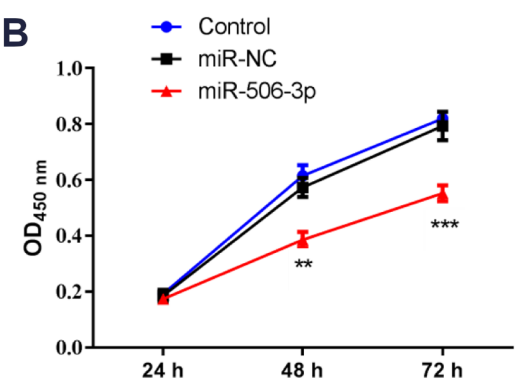

miR-506-3p

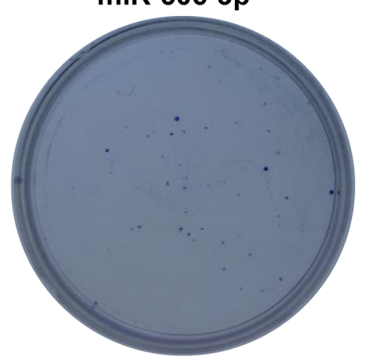

miR-506-3p

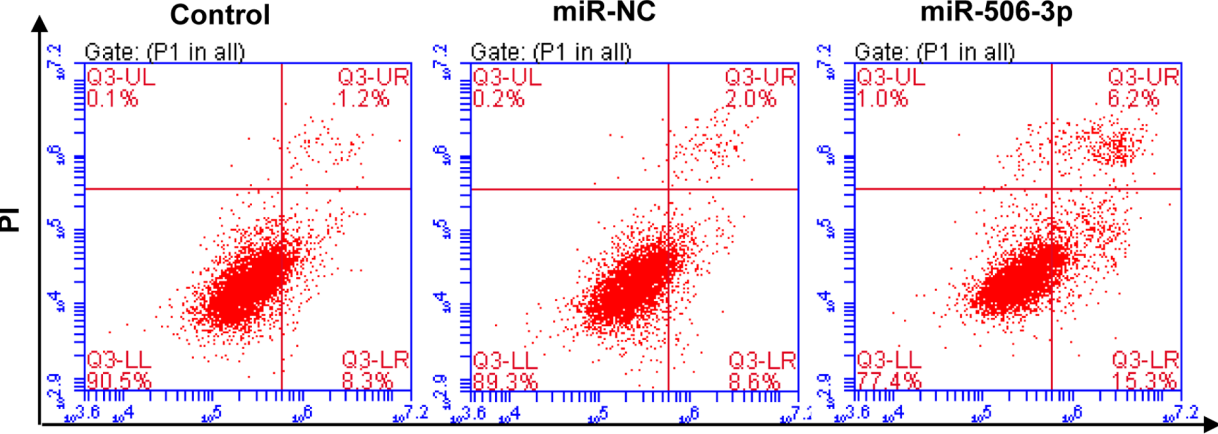

Annexin V FITC

E

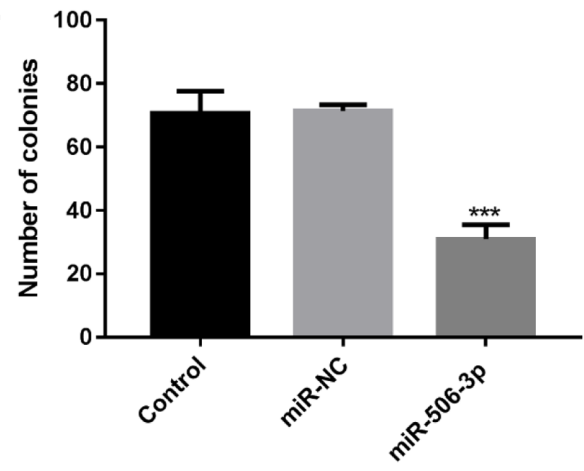

$\mathbf{F}$

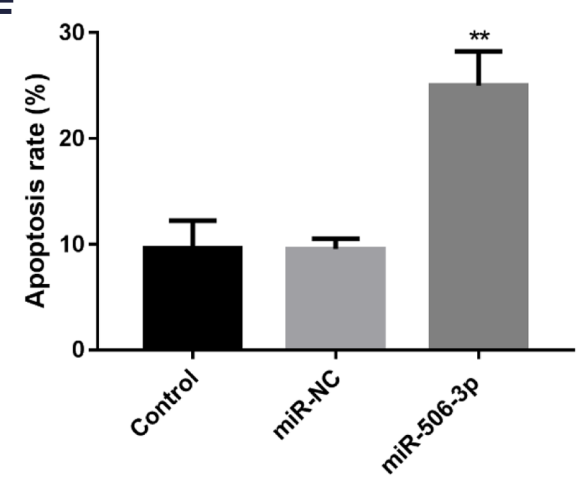

Figure 2. MiR-506-3p inhibited cell proliferation and induced cell apoptosis of OC. A) Transfection efficiency of miR-506-3p mimics via qRT-PCR. B) MiR-506-3p inhibited the cell viability of HO-8910PM cells. C) MiR-506-3p inhibited the clone formation ability of HO-8910PM cells. D) MiR-506-3p induced the cell apoptosis of HO-8910PM cells. E) The number of colonies was quantitated by miR-506-3p mimics in HO-8910PM cells. F) The apoptosis rate was quantitated by miR-506-3p mimics in HO-8910PM cells. ${ }^{\star *} \mathrm{p}<0.01,{ }^{* *} \mathrm{p}<0.01$ represents comparison with miR-NC.

miR-506-3p might bind to 3'UTR of SIRT1 in OC. Secondly, the expression of SIRT1 was inhibited by miR-506-3p mimics (Figure 3C), revealing the negative correlation between them. Lastly, western blot analysis revealed that miR-506-3p decreased the expression of SIRT1 (Figures 3D and 3E). Although the downstream targets of SIRT1 such as AKT and FOXO3a were not affected by miR-506-3p, the p-AKT and $\mathrm{p}-\mathrm{FOXO} 3 \mathrm{a}$ were decreased under the condition of 
miR-506-3p overexpression (Figures 3D and 3E), suggesting the relation between miR-506-3p and SIRT1/AKT/FOXO3a signaling pathway.

Overexpression SIRT1 promoted cell proliferation and inhibited cell apoptosis of OC. We have demonstrated that SIRT1 was a direct target gene of miR-506-3p in OC. Therefore, we verified the effect of SIRT1 to the expression of miR-506-3p. HO-8910PM cells transfected with Vector and pcDNA3.1-SIRT1 was established, respectively. Firstly, qRT-PCR analysis revealed that the expression of SIRT1 was significantly upregulated in SIRT1 overexpression group than that in control with Vector group while the expression of miR-506-3p was significantly downregulated (Figures 4A and 4B). Secondly, CCK-8 (Figure 4C) and colony formation assays (Figure 4D) showed that the cell viability and cell prolif- eration were significantly promoted by addition of SIRT1 overexpression. The number of colonies of HO-8910PM cells transfected with pcDNA3.1-SIRT1 was dramatically increased compared to cells in control with Vector group (Figure 4E). Lastly, flow cytometry also confirmed that cell apoptosis was inhibited by addition of SIRT1 overexpression (Figure 4F and 4G). Moreover, the increased SIRT1, p-AKT and p-FOXO3a expression were also observed in SIRT1 overexpression group compared to control with Vector group (Figures $4 \mathrm{H}$ and $4 \mathrm{I}$ ). In conclusion, these results demonstrated that overexpression SIRT1 promoted cell proliferation and inhibited cell apoptosis of OC.

MiR-506-3p inhibited cell proliferation and induced cell apoptosis of OC via SIRT1. It was suggested that SIRT1 may be related to miR-506-3p mediated suppression of OC

A

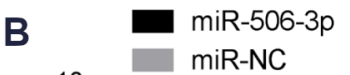

SIRT1 3'UTR 5' UUUAAAAGCUUAGCCUGCCUUAA 3'

Has-miR-506-3p 3' AGAUGAGUCUUCCCACGGAAUG 5'

C

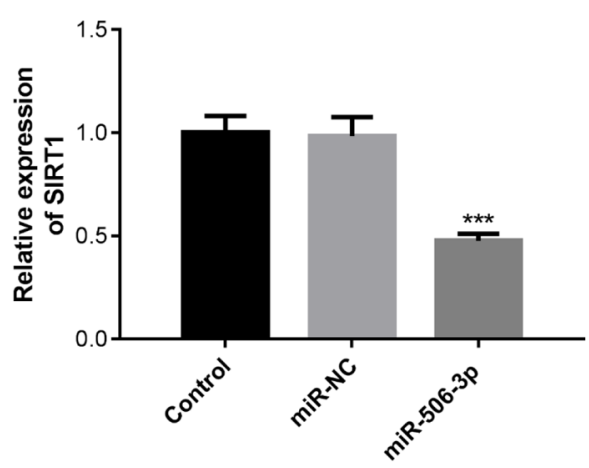

E

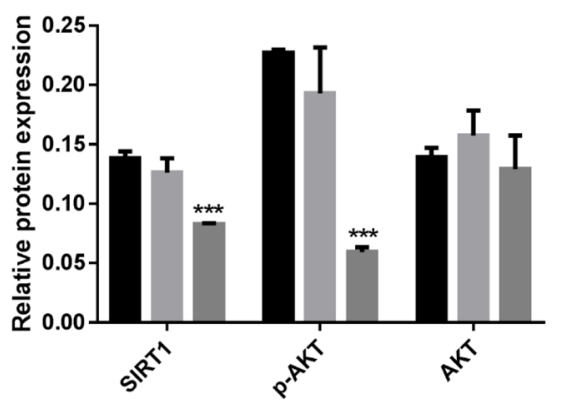

D
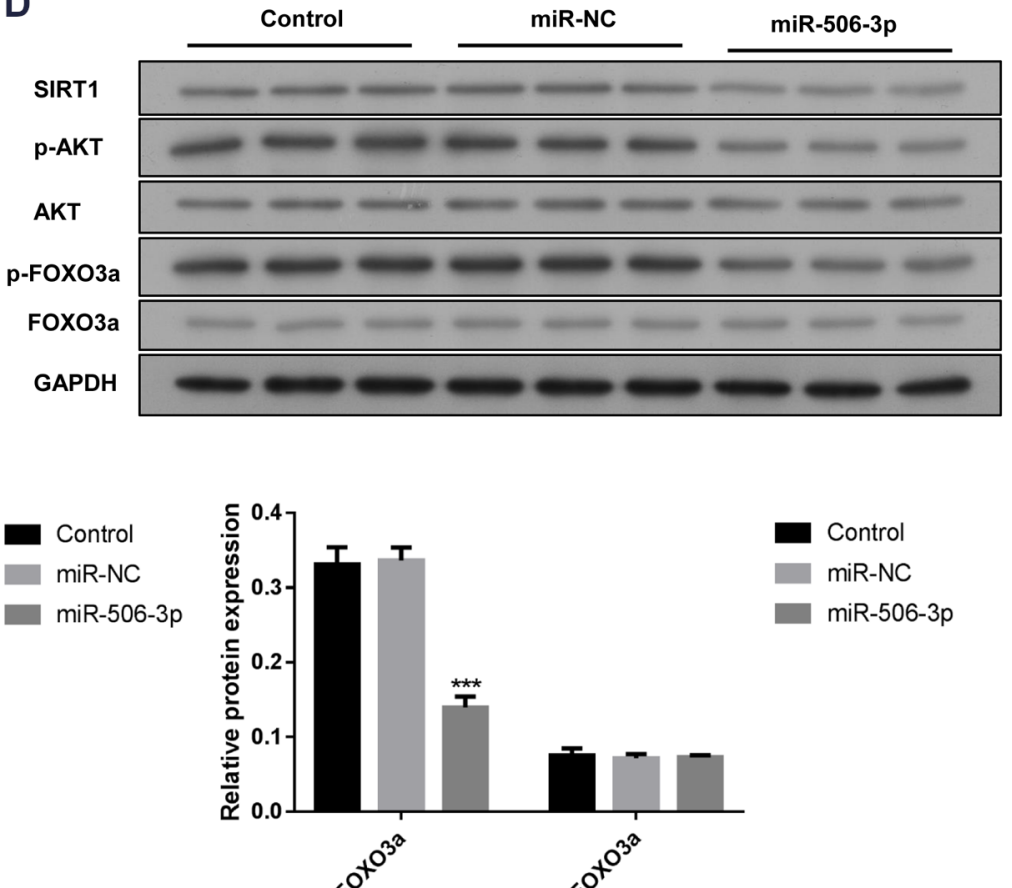

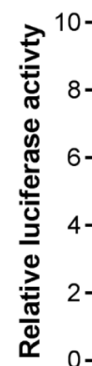

miR-NC

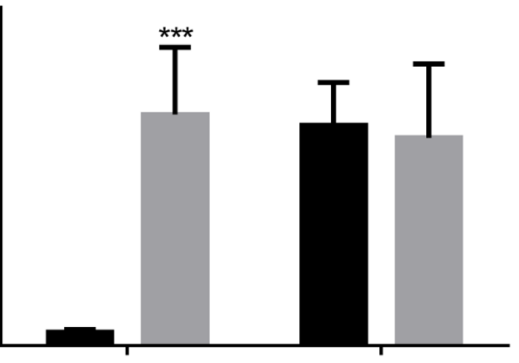

SIRT1-W

SIRT1-MUT 
A

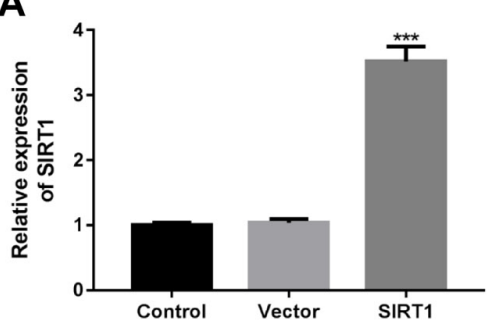

D

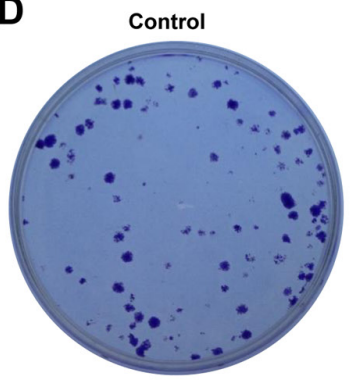

F
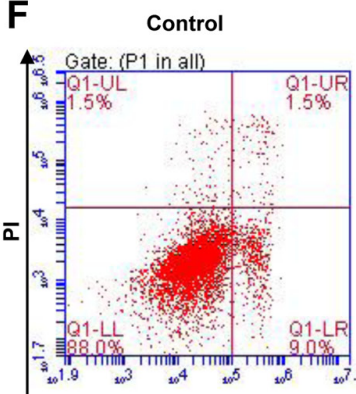

B
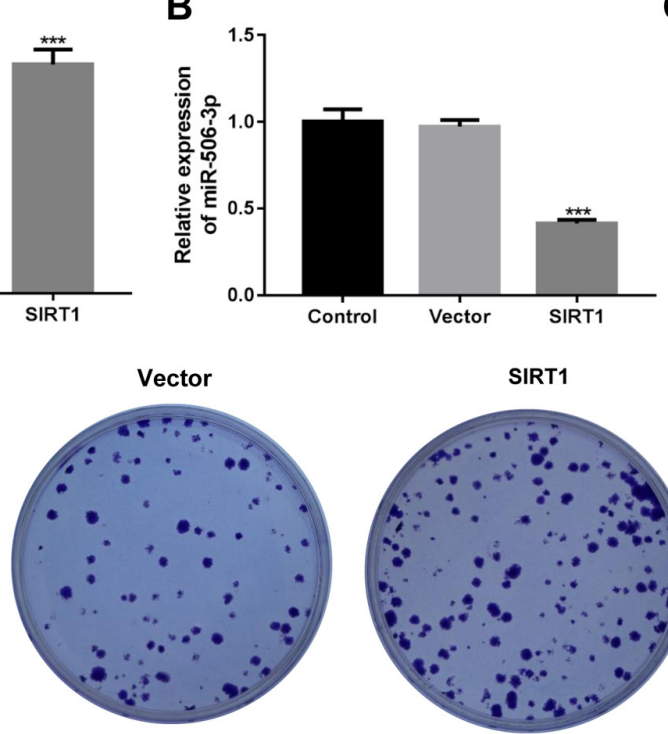

Vector

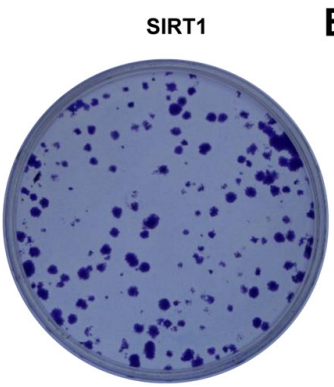

SIRT1

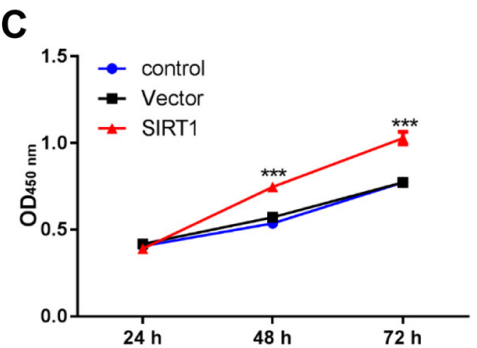

E

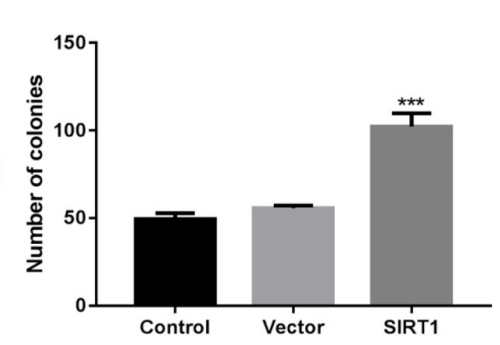

G

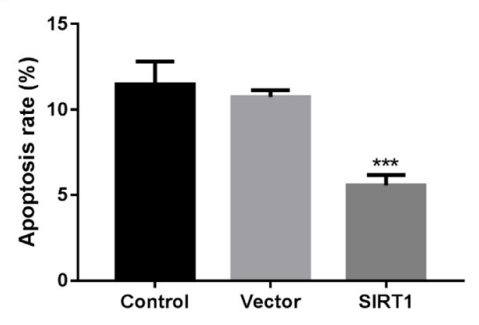

H

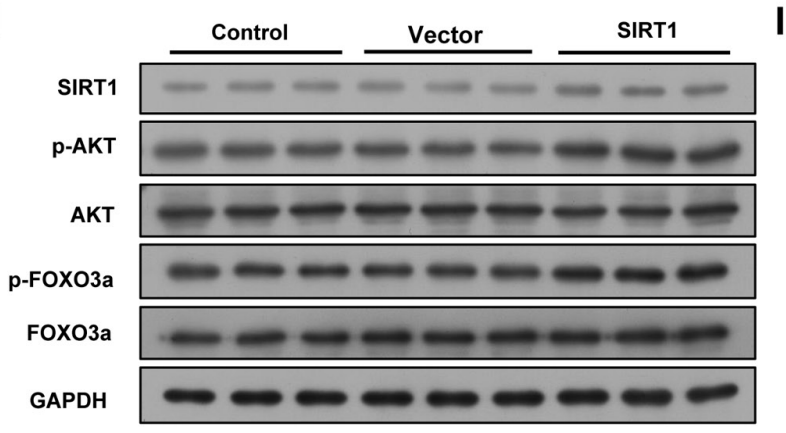

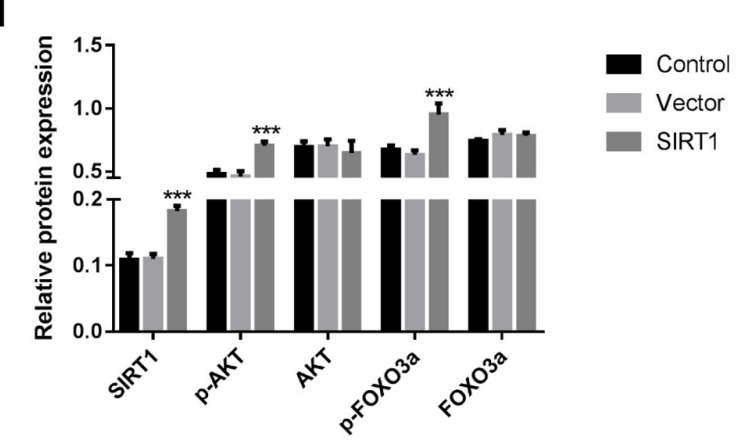

Figure 4. Overexpression SIRT1 promoted cell proliferation and inhibited cell apoptosis of OC. A) qRT-PCR analysis of SIRT1 in HO-8910PM cells transfected with control vector and pcDNA3.1-SIRT1, respectively. B) qRT-PCR analysis of miR-506-3p in HO-8910PM cells transfected with Vector or pcDNA3.1-SIRT1, respectively. C) Cell viability of HO-8910PM cell was increased by addition with SIRT1. D) Effect of SIRT1 on the cell proliferation of HO-8910PM cells by colony formation assay. E) The number of colonies in HO-8910PM cells transfected with vector or pcDNA3.1SIRT1 were counted, respectively. F) Flow cytometry showed the inhibition ability of SIRT1 on cell apoptosis. G) The apoptosis rate of HO-8910PM cells transfected with Vector or pcDNA3.1-SIRT1, respectively. H) Western blot analysis of expression of SIRT1, AKT, p-AKT, FOXO3a, p-FOXO3a affected by SIRT1. I) The relative protein expression of SIRT1, AKT, p-AKT, FOXO3a, p-FOXO3a was calculated by ImageJ. ${ }^{\star * *}$ p $<0.001$ represents comparison with Vector.

cell proliferation and promotion of cell apoptosis based on the aforementioned results. We then decided to investigate this speculation. Stable HO-8910PM cell line transfected with miR-506-3p mimics or co-transfected with miR-506-3p mimics and pcDNA3.1-SIRT1 was established. Firstly, qRT-PCR analysis revealed that the expression of miR-506-3p induced by miR-506-3p mimics was downregulated by the addition of SIRT1 overexpression (Figure 5A); on the other 
A

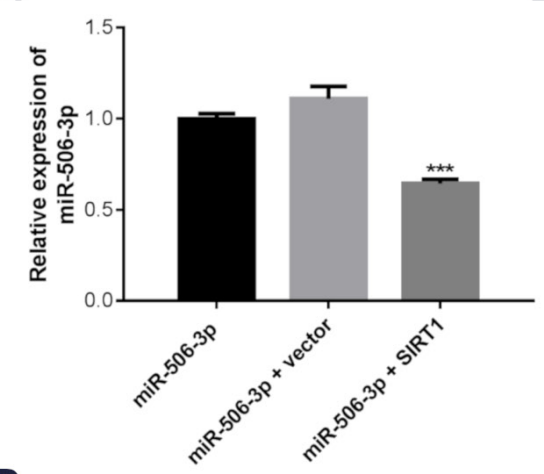

D

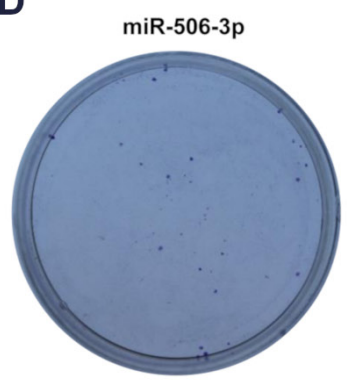

F

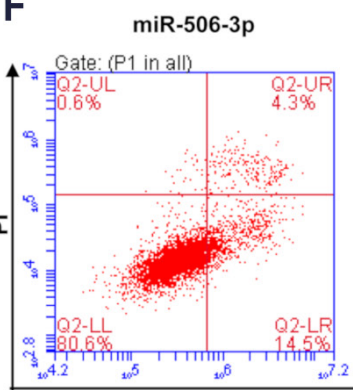

B

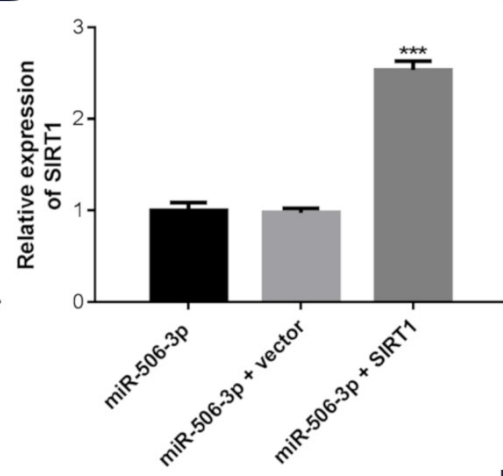

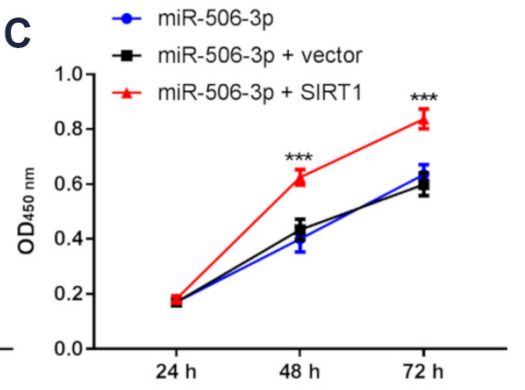

E

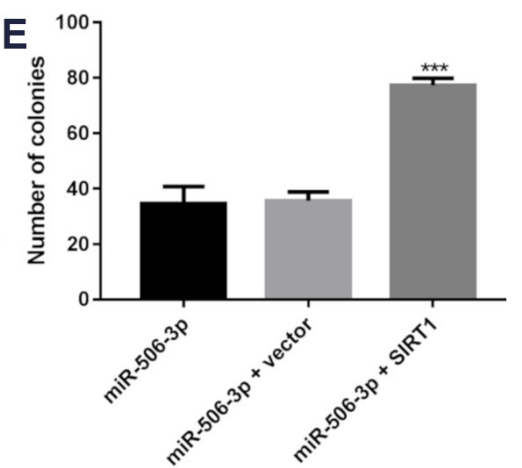

H miR-506-3p + Vector miR-506-3p + SIRT1
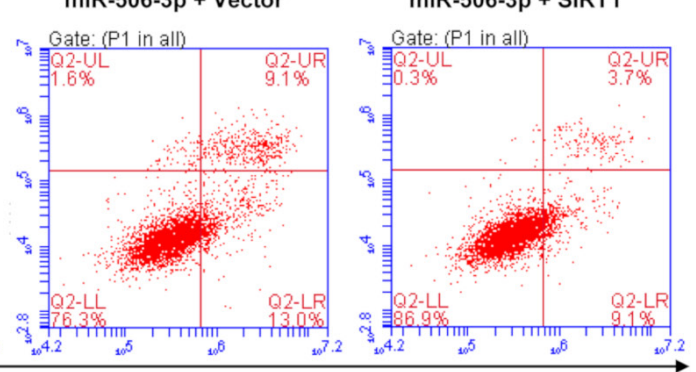

miR-506-3p + SIRT1
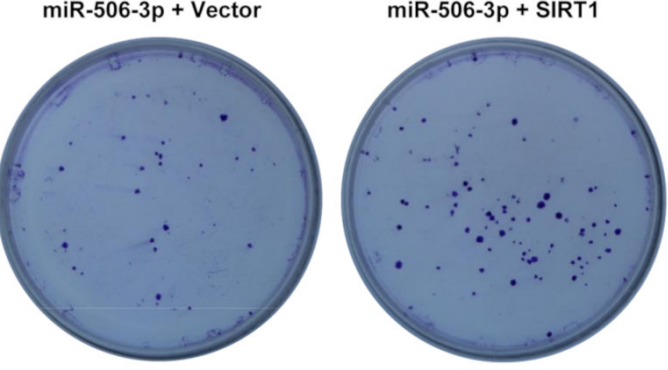

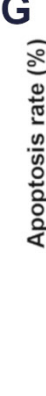

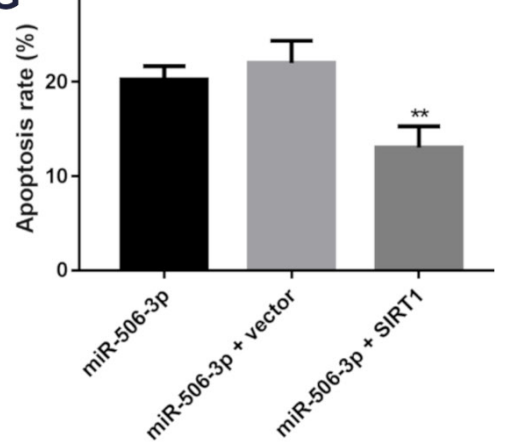

Annexin V FITC
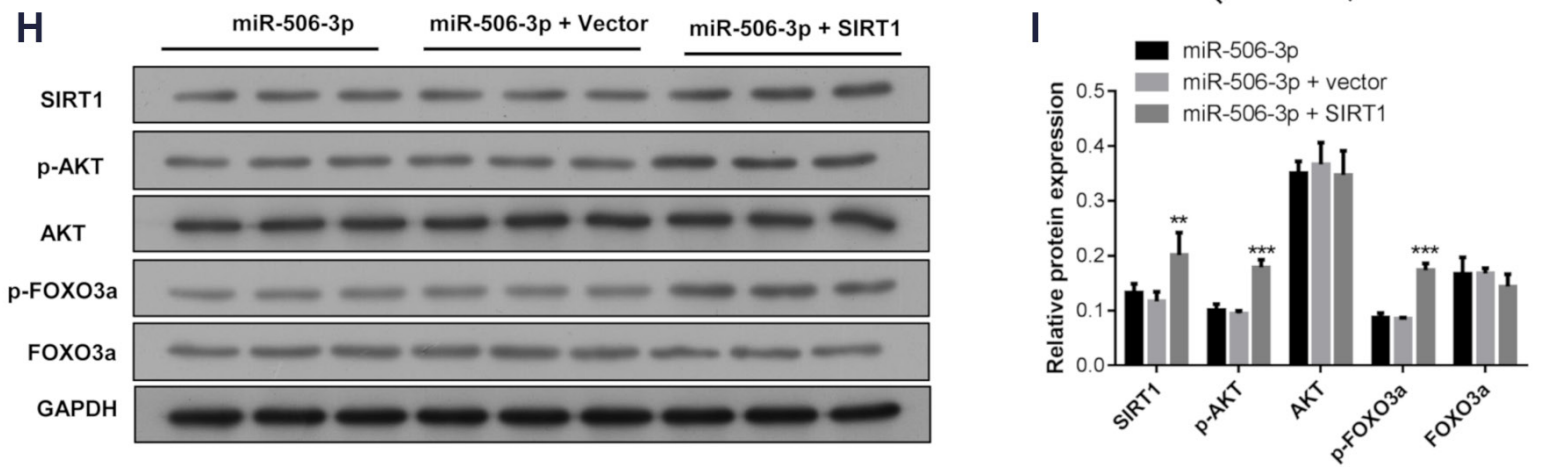

Figure 5. MiR-506-3p inhibited cell proliferation and induced cell apoptosis of OC via SIRT1. A) qRT-PCR analysis of miR-506-3p in HO-8910PM cells transfected with miR-506-3p mimics alone or co-transfected with miR-506-3p mimics and pcDNA3.1-SIRT1. B) qRT-PCR analysis of SIRT1 in HO-8910PM cells transfected with miR-506-3p mimics alone or co-transfected with miR-506-3p mimics and pcDNA3.1-SIRT1. C) Cell viability of HO-8910PM cell was decreased by miR-506-3p mimics, while addition of SIRT1 increased the cell viability. D) Effect of miR-506-3p and SIRT1 on the cell proliferation of HO-8910PM cells by colony formation assay. E) The number of colonies in HO-8910PM cells transfected with miR-506-3p mimics alone or co-transfected with miR-506-3p mimics and pcDNA3.1-SIRT1 were counted. F) Flow cytometry showed the promotion ability of miR-506-3p on cell apoptosis reversed by addition of SIRT1. G) The apoptosis rate of HO-8910PM cells transfected with miR-506-3p mimics alone or co-transfected with miR-506-3p mimics and pcDNA3.1-SIRT1 were counted. H) Western blot analysis of expression of SIRT1, AKT, p-AKT, FOXO3a, p-FOXO3a affected by miR-506-3p mimics and SIRT1. I) The relative protein expression of SIRT1, AKT, p-AKT, FOXO3a, p-FOXO3a were quantified. ${ }^{* *}$ p $<0.01$, ${ }^{* * *} \mathbf{p}<0.001$ represents comparison with miR-506-3p mimics + Vector. 
hand, the decreased expression of SIRT1 by miR-506-3p was also upregulated by addition of SIRT1 overexpression (Figure 5B). Secondly, CCK-8 (Figure 5C) and colony formation assays (Figure 5D) showed that the cell viability and cell proliferation inhibited by miR-506-3p mimics were promoted by addition of SIRT1 overexpression. The number of colonies of HO-8910PM cells co-transfected with miR-506-3p mimics and pcDNA3.1-SIRT1 was dramatically increased compared to cells transfected with miR-506-3p mimics (Figure 5E). Lastly, flow cytometry also confirmed that cell apoptosis promoted by miR-506-3p mimics was also inhibited by addition of SIRT 1 overexpression (Figures $5 \mathrm{~F}$ and $5 \mathrm{G})$. Moreover, the decreased p-AKT and p-FOXO3a expression under the condition of miR-506-3p mimics were also increased by addition of SIRT1 overexpression (Figures $5 \mathrm{H}$ and 5I). In conclusion, these results demonstrated that the regulation of miR-506-3p on OC proliferation and apoptosis was partially through suppression of SIRT1.

\section{Discussion}

It has been well studied that the development of OC is closely associated with the abnormal cell proliferation and apoptosis [28]. Based on this, the screening for proteins or other molecules related to the inhibition of OC cell proliferation or promotion of cell apoptosis are crucial for the novel therapeutic schedules to treat OC [29-33]. Previous study has shown that in cervical cancer, miR-506 functioned as a tumor suppressor via inhibition of cell proliferation and promotion of cell apoptosis [34]. However, the underlying regulatory mechanism of miR-506-3p on OC progression remains largely unclear. Our current study then investigated the effects and underlying mechanisms of miR-506-3p on OC cell proliferation and apoptosis. We provided a proof of concept that miR-506-3p repressed proliferation and promoted apoptosis of OC cells, at least partially via inhibition of SIRT1 expression.

MiR-506-3p was shown to be downregulated in OC tissues compared with normal ovarian tissue [35], that is also confirmed in the current study. Moreover, miR-506-3p was robustly decreased in recurrent serous OC compared with primary OC tissues, consistent with the previous study [35], suggesting that the decreased expression of miR-506-3p was dramatically associated with advanced OC malignancy. Through regulation of vimentin or N-cadherin, miR-506 inhibited cell migration and invasion, thus associating with prognosis in epithelial OC [36]. MiR-506 was also involved in the regulation network of long noncoding RNA MALAT1/iASPP (inhibitor of apoptosis stimulating protein of p53) axis on OC growth [37]. Therefore, the miR-506-3p anti-proliferation and apoptotic signaling were found to be involved in the inhibition of tumor growth in OC $[38,39]$. Especially, recent study showed that through directly targeting CDK4/6-FOXM1 axis, miR-506 inhibited cell proliferation and growth of OC [17]. Consistent with these researches, miR-506-3p inhibited cell proliferation and induced cell apoptosis in OC. Moreover, the potential target of miR-506-3p, SIRT1 was found and confirmed by luciferase reporter assay. The directly binding and negatively regulation of miR-506-3p on SIRT1 was firstly demonstrated in the present study.

SIRT1 was shown to be a target gene of various miRNAs in a variety of tumors [40-42]. SIRT1 regulated invasiveness of ovarian carcinoma cells [26] and OC progression [43], and overexpression of SIRT1 resulted in poor prognosis in serous epithelial OC [25]. Similarly, miR-142-3p was found to inhibit cell proliferation of OC via targeting SIRT1 [44]. However, the downstream targets of SIRT1 involved in OC progression remain unclear. In the present study, we firstly demonstrated that overexpression SIRT1 promoted cell proliferation and inhibited cell apoptosis of OC, suggesting the important role of SIRT1 in OC. Furthermore, we found that AKT and FOXO3a were the potential downstream targets of SIRT1. MiR-506-3p had no significant effect on AKT and FOXO3a expression, but decreased $\mathrm{p}-\mathrm{AKT}$ and $\mathrm{p}$-FOXO3a expression in OC cell lines. Moreover, addition of SIRT1 overexpression could reverse the inhibition ability of miR-506-3p on p-AKT and p-FOXO3a expression, confirming that SIRT1/ AKT/FOXO3a network was involved in the regulation of miR-506-3p on OC progression. SIRT1 was shown to increase the expression of $\mathrm{p}$-AKT and promote AKT activity, thus facilitating for cell proliferation and preventing cell apoptosis of breast cancer [45]. Moreover, FOXO3 was considered as an important tumor suppressor in a variety of cancers [46], while AKT phosphorylated FOXO3a and thus inactivating this transcription factor [47]. However, due to the complicated downstream targets of SIRT1, other signaling pathways involved in the regulation of miR-506-3p on OC need further to be investigated.

In summary, the present study demonstrated that miR-506-3p not only inhibited cell proliferation, but also promoted cell apoptosis in OC via negative regulation of SIRT1, thus inhibiting OC progression. This finding illuminated the relation between miR-506-3p/SIRT1/AKT/ FOXO3a regulatory axis and $\mathrm{OC}$ cell progression, suggesting potential application of miR-506-3p in treatment for OC.

Acknowledgements: This research was funded by the Scientific Research Project of Human Health and Family Planning Commission in 2017.

\section{References}

[1] SIEGEL RL, MILLER KD, JEMAL A. Cancer statistics, 2015. CA Cancer J Clin 2015; 65: 5-29. https://doi.org/10.3322/ caac. 21254

[2] ARMBRUSTER S, COLEMAN RL, RAUH-HAIN JA. Management and Treatment of Recurrent Epithelial Ovarian Cancer. Hematol Oncol Clin North Am 2018; 32: 965-982. https://doi.org/10.1016/j.hoc.2018.07.005 
[3] MCGUIRE WP, HOSKINS WJ, BRADY MF, KUCERA PR, PARTRIDGE EE et al. Cyclophosphamide and cisplatin compared with paclitaxel and cisplatin in patients with stage III and stage IV ovarian cancer. N Engl J Med 1996; 334: 1-6. https://doi.org/10.1056/NEJM199601043340101

[4] TORRE LA, TRABERT B, DESANTIS CE, MILLER KD, SAMIMI G et al. Ovarian cancer statistics, 2018. CA Cancer J Clin 2018; 68: 284-296. https://doi.org/10.3322/caac.21456

[5] SIEGEL RL, MILLER KD, JEMAL A. Cancer statistics, 2018. CA Cancer J Clin 2018; 68: 7-30. https://doi.org/10.3322/ caac. 21442

[6] MIAO Y, LU M, YAN Q, LI S, FENG. Inhibition of Proliferation, Migration, and Invasion by Knockdown of Pyruvate Kinase-M2 (PKM2) in Ovarian Cancer SKOV3 and OVCAR3 Cells Oncol Res 2016; 24: 463-475. https://doi.org/10.3727/0 96504016X14685034103671

[7] TENG Y, ZUO X, HOU M, ZHANG Y, LI C et al. A Double-Negative Feedback Interaction between MicroRNA-29b and DNMT3A/3B Contributes to Ovarian Cancer Progression Cell Physiol Biochem 2016; 39: 2341-2352. https://doi. org/10.1159/000447926

[8] SUN K, LAI EC. Adult-specific functions of animal microRNAs. Nat Rev Genet 2013; 14: 535-548. https://doi. org/10.1038/nrg3471

[9] BARTEL DP. MicroRNAs: genomics, biogenesis, mechanism, and function. Cell 2004; 116: 281-297. https://doi. org/10.1016/s0092-8674(04)00045-5

[10] ASAMA H, SUZUKI R, HIKICHI T, TAKAGI T, MASAMUNE A et al. MicroRNA let-7d targets thrombospondin-1 and inhibits the activation of human pancreatic stellate cells. Pancreatology 2019; 19: 196-203. https://doi.org/10.1016/j. pan.2018.10.012

[11] YANG B, XIE R, WU SN, GAO CC, YANG XZ ET AL. MicroRNA-615-5p targets insulin-like growth factor 2 and exerts tumor-suppressing functions in human esophageal squamous cell carcinoma. Oncol Rep 2018; 39: 255-263. https://doi.org/10.3892/or.2017.6079

[12] JOHN B, ENRIGHT AJ, ARAVIN A, TUSCHL T, SANDER C et al. Human MicroRNA targets. PLoS Biol 2004; 2: e363. HTTPS://DOI.ORG/10.1371/journal.pbio.0020363

[13] CALIN GA, CROCE CM. MicroRNA signatures in human cancers. Nat Rev Cancer 2006; 6: 857-866. https://doi. org/10.1038/nrc1997

[14] HAYES J, PERUZZI PP, LAWLER S. MicroRNAs in cancer: biomarkers, functions and therapy. Trends Mol Med 2014; 20: 460-469. https://doi.org/10.1016/j.molmed.2014.06.005

[15] LOU Y, YANG X, WANG F, CUI Z, HUANG Y. MicroRNA-21 promotes the cell proliferation, invasion and migration abilities in ovarian epithelial carcinomas through inhibiting the expression of PTEN protein. Int J Mol Med 2010; 26: 819-827. https://doi.org/10.3892/ijmm_00000530

[16] PARIKH A, LEE C, JOSEPH P, MARCHINI S, BACCARINI A et al. microRNA-181a has a critical role in ovarian cancer progression through the regulation of the epithelial-mesenchymal transition. Nat Commun 2014; 5: 2977. https://doi. org/10.1038/ncomms3977
[17] LIU G, SUN Y, JI P, LI X, COGDELL D et al. MiR-506 suppresses proliferation and induces senescence by directly targeting the CDK4/6-FOXM1 axis in ovarian cancer. J Pathol 2014; 233: 308-318. https://doi.org/10.1002/path.4348

[18] LIU MX, SIU MK, LIU SS, YAM JW, NGAN HY et al. Epigenetic silencing of microRNA-199b-5p is associated with acquired chemoresistance via activation of JAG1-Notch1 signaling in ovarian cancer. Oncotarget 2014; 5: 944-958. https://doi.org/10.18632/oncotarget.1458

[19] JIASHI W, CHUANG Q, ZHENJUN Z, GUANGBIN W, BIN L et al. MicroRNA-506-3p inhibits osteosarcoma cell proliferation and metastasis by suppressing RAB3D expression. Aging 2018; 10: 1294-1305. https://doi.org/10.18632/ aging. 101468

[20] WANG Y, JIAQI C, ZHAOYING C, HUIMIN C. MicroRNA506-3p regulates neural stem cell proliferation and differentiation through targeting TCF3. Gene 2016; 593: 193-200. https://doi.org/10.1016/j.gene.2016.08.026

[21] WANG L, ZHANG Z, YU X, HUANG X, LIU Z et al. Unbalanced YAP-SOX9 circuit drives stemness and malignant progression in esophageal squamous cell carcinoma. Oncogene 2019; 38: 2042-2055. https://doi.org/10.1038/s41388-0180476-9

[22] GUO S, YANG P, JIANG X, LI X, WANG Y et al. Genetic and epigenetic silencing of mircoRNA-506-3p enhances COTL1 oncogene expression to foster non-small lung cancer progression. Oncotarget 2017; 8: 644-657. https://doi. org/10.18632/oncotarget.13501

[23] CANTO C, AUWERX J. PGC-1alpha, SIRT1 and AMPK, an energy sensing network that controls energy expenditure. Curr Opin Lipidol 2009; 20: 98-105. https://doi.org/10.1097/ MOL.0b013e328328d0a4

[24] YI J, LUO J. SIRT1 and p53, effect on cancer, senescence and beyond. Biochim Biophys Acta 2010; 1804: 1684-1689. https://doi.org/10.1016/j.bbapap.2010.05.002

[25] SHUANG T, WANG M, ZHOU Y, SHI C. Over-expression of Sirt1 contributes to chemoresistance and indicates poor prognosis in serous epithelial ovarian cancer (EOC). Med Oncol 2015; 32: 260. https://doi.org/10.1007/s12032-0150706-8

[26] MVUNTA DH, MIYAMOTO T, ASAKA R, YAMADA Y, ANDO $\mathrm{H}$ et al. SIRT1 Regulates the Chemoresistance and Invasiveness of Ovarian Carcinoma Cells. Transl Oncol 2017; 10: 621-631. https://doi.org/10.1016/j.tranon.2017.05.005

[27] LIVAK KJ, SCHMITTGEN TD. Analysis of relative gene expression data using real-time quantitative PCR and the 2(-Delta Delta C(T)) Method. Methods 2001; 25: 402-408. https://doi.org/10.1006/meth.2001.1262

[28] LENGYEL E. Ovarian cancer development and metastasis. Am J Pathol 2010; 177: 1053-1064. https://doi.org/10.2353/ ajpath.2010.100105

[29] GAO Y, FOSTER R, YANG X, FENG Y, SHEN JK et al. Up-regulation of CD44 in the development of metastasis, recurrence and drug resistance of ovarian cancer. Oncotarget 2015; 6: 9313-9326. https://doi.org/10.18632/oncotarget. 3220 
[30] LOGINOV VI, BURDENNYY AM, FILIPPOVA EA, PRONINA IV, KAZUBSKAYA TP et al. [Hypermethylation of miR-107, miR-130b, miR-203a, miR-1258 Genes Associated with Ovarian Cancer Development and Metastasis]. Mol Biol (Mosk) 2018; 52: 801-809. https://doi.org/10.1134/ S0026898418050105

[31] SAVANT SS, SRIRAMKUMAR S, O'HAGAN HM. The Role of Inflammation and Inflammatory Mediators in the Development, Progression, Metastasis, and Chemoresistance of Epithelial Ovarian Cancer. Cancers 2018; 10: E251. https:// doi.org/10.3390/cancers10080251

[32] WU B, LI S, SHENG L, ZHU J, GU L et al. Metformin inhibits the development and metastasis of ovarian cancer. Oncol Rep 2012; 28: 903-908. https://doi.org/10.3892/ or.2012.1890

[33] XIONG X, ZHANG J, HUA X, CAO W, QIN S et al. FBP1 promotes ovarian cancer development through the acceleration of cell cycle transition and metastasis. Oncol Lett 2018; 16: 1682-1688. https://doi.org/10.3892/ol.2018.8872

[34] WEN SY, LIN Y, YU YQ, CAO SJ, ZHANG R et al. miR-506 acts as a tumor suppressor by directly targeting the hedgehog pathway transcription factor Gli3 in human cervical cancer. Oncogene 2015; 34: 717-725. https://doi.org/10.1038/ onc.2014.9

[35] NAM EJ, KIM S, LEE TS, KIM HJ, LEE JY et al. Primary and recurrent ovarian high-grade serous carcinomas display similar microRNA expression patterns relative to those of normal ovarian tissue. Oncotarget 2016; 7: 70524-70534. https://doi.org/10.18632/oncotarget.12045

[36] SUN Y, HU L, ZHENG H, BAGNOLI M, GUO Y et al. MiR506 inhibits multiple targets in the epithelial-to-mesenchymal transition network and is associated with good prognosis in epithelial ovarian cancer. J Pathol 2015; 235: 25-36. https://doi.org/10.1002/path.4443

[37] LEI R, XUE M, ZHANG L, LIN Z. Long noncoding RNA MALAT1-regulated microRNA 506 modulates ovarian cancer growth by targeting iASPP. Onco Targets Ther 2016; 10: 35-46. https://doi.org/10.2147/OTT.S112686
[38] LIU G, YANG D, RUPAIMOOLE R, PECOT CV, SUN Y et al. Augmentation of response to chemotherapy by microRNA-506 through regulation of RAD51 in serous ovarian cancers. J Natl Cancer Inst 2015; 107: djv108. https://doi. org/10.1093/jnci/djv108

[39] LIU G, XUE F, ZHANG W. miR-506: a regulator of chemosensitivity through suppression of the RAD51-homologous recombination axis. Chin J Cancer 2015; 34: 485-487. https://doi.org/10.1186/s40880-015-0049-Z

[40] YE Z, FANG J, DAI S, WANG Y, FU Z et al. MicroRNA$34 \mathrm{a}$ induces a senescence-like change via the down-regulation of SIRT1 and up-regulation of p53 protein in human esophageal squamous cancer cells with a wild-type p53 gene background. Cancer Lett 2016; 370: 216-221. https://doi. org/10.1016/j.canlet.2015.10.023

[41] GAO J, WANG Y, ZHAO X, CHEN P, XIE L. MicroRNA204-5p-Mediated Regulation of SIRT1 Contributes to the Delay of Epithelial Cell Cycle Traversal in Diabetic Corneas. Invest Ophthalmol Vis Sci 2015; 56: 1493-1504. https://doi. org/10.1167/iovs.14-15913

[42] YAMAKUCHI M. MicroRNA Regulation of SIRT1. Front Physiol 2012; 3: 68. https://doi.org/10.3389/fphys.2012.00068

[43] LI D, BI FF, CHEN NN, CAO JM, SUN WP et al. A novel crosstalk between BRCA1 and sirtuin 1 in ovarian cancer. Sci Rep 2014; 4: 6666. https://doi.org/10.1038/srep06666

[44] GAO J, WU N, LIU X, XIA Y, CHEN Y et al. MicroRNA$142-3 p$ inhibits cell proliferation and chemoresistance in ovarian cancer via targeting sirtuin 1. Exp Ther Med 2018; 15: 5205-5214. https://doi.org/10.3892/etm.2018.6107

[45] JIN X, WEI Y, XU F, ZHAO M, DAI K et al. SIRT1 promotes formation of breast cancer through modulating Akt activity. J Cancer 2018; 9: 2012-2023. https://doi.org/10.7150/ jca. 24275

[46] ZHANG X, TANG N, HADDEN TJ, RISHI AK. Akt, FoxO and regulation of apoptosis. Biochim Biophys Acta 2011; 1813: 1978-1986. https://doi.org/10.1016/j.bbamcr.2011.03.010

[47] LIU J, DUAN Z, GUO W, ZENG L, WU Y et al. Targeting the BRD4/FOXO3a/CDK6 axis sensitizes AKT inhibition in luminal breast cancer. Nat Commun 2018; 9: 5200. https:// doi.org/10.1038/s41467-018-07258-y 